\title{
MS34-P07 | DeVelopment of ACcurate ANd EfFicient AB initio Potentials for Effective Crystal Structure Prediction
}

Bowskill, David (Imperial College London, London, GBR); Sugden, Isaac (Imperial College London, London, GBR); Pantelides, Constantinos (Imperial College London, London, GBR); Adjiman, Claire (Imperial College London, London, GBR)

Recent blind tests organised by the CCDC have highlighted the growing capabilities of crystal structure prediction (CSP) for increasingly complex systems, but what is often considered a success in a blind test does not always provide sufficient information to complement experimental solid-form screening efforts in an effective way. Despite the requirement for efficient methods, the use of computationally expensive periodic density functional theory (DFT) calculations has become a staple for many practitioners of CSP. Although this typically achieves greater accuracy, the computational cost may often be prohibitively large for the study of systems of industrial relevance particularly in the early stages of product development.

In this study, we investigate the role of parameter estimation and adaptive force fields in the development of bespoke $a b$ initio potentials. The proposed methodology aims to improve the reliability of existing force field models used commonly in CSP to be competitive in accuracy with much more expensive DFT calculations. These improvements are facilitated through the rigorous construction of training sets from theoretical calculations. The application of these training sets vastly increases the abundance of available data. In addition, comparison with experimentally derived training sets demonstrates large discrepancies which can mainly be attributed to high experimental uncertainties and mischaracterised free energy corrections. Beyond this, by taking cues from successful developments in DFT dispersion corrections over recent years, we aim to use these training sets to develop tailored $a b$ initio potentials that incorporate the effects of the molecular environment into the potential seamlessly whilst retaining parameter transferability. 